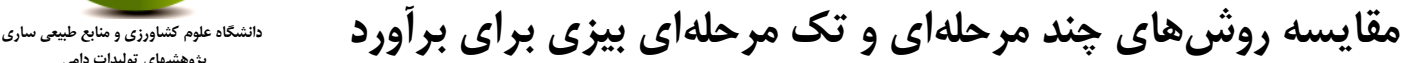

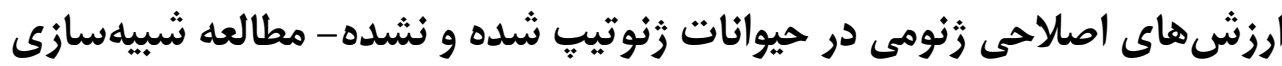

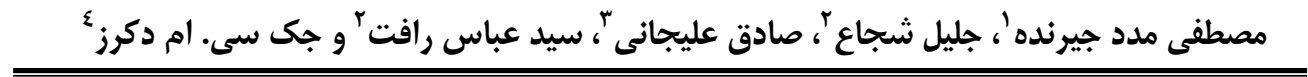

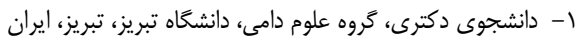

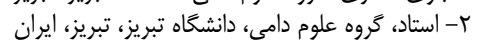

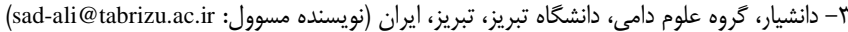

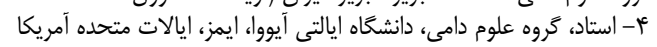

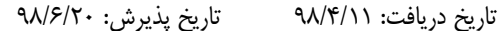

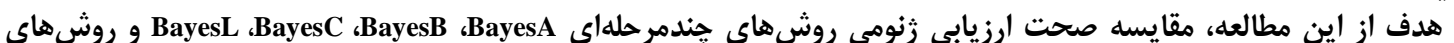

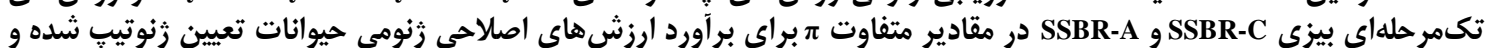

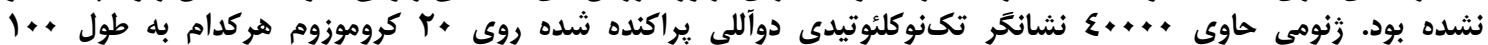

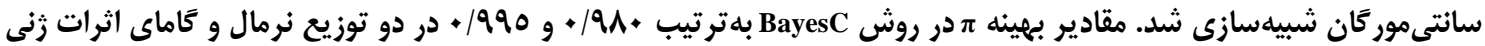

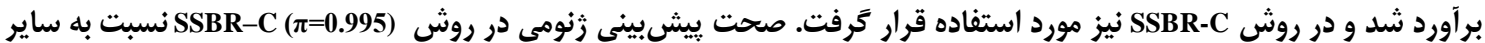

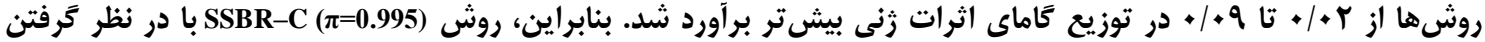

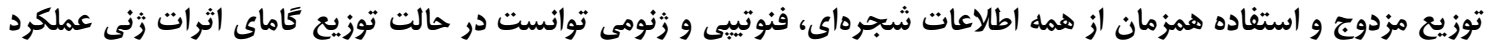

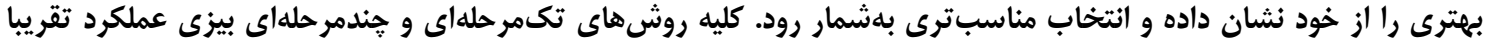

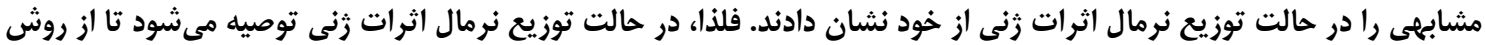

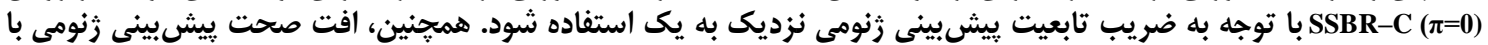

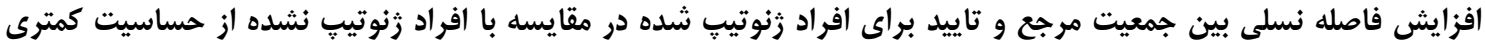
برخوردار بود. وازههاى كليدى: انتخاب زنومى، بيزى، روش خُندمر حلهاى، شبيهسازى، صحت زنومى

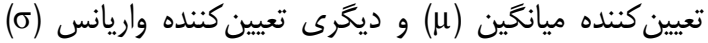

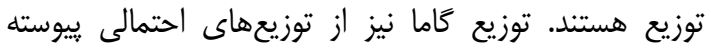

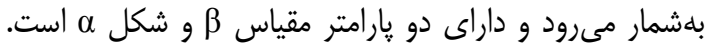

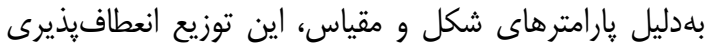
زيادى براى تحليل هر مجموعه داده حقيقى مثبت را را داراست

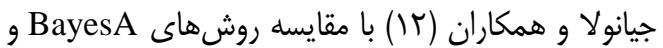

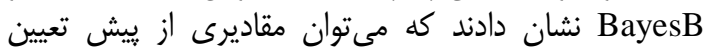

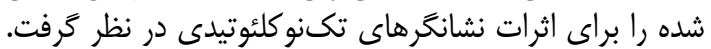

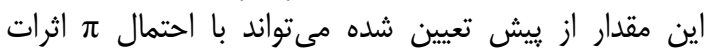

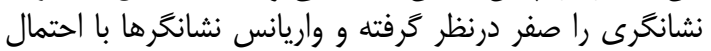

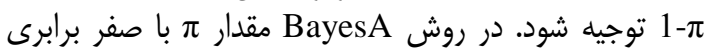

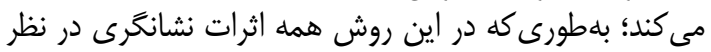

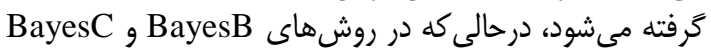

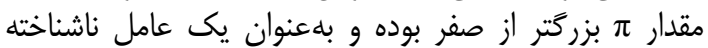

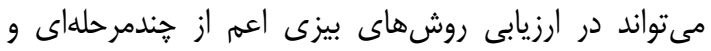

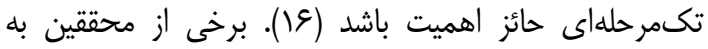

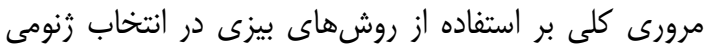

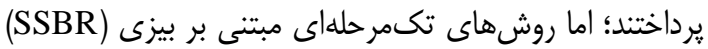

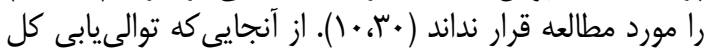

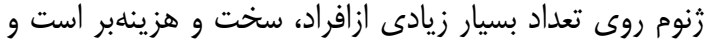

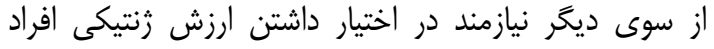

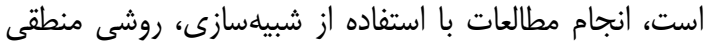
براى جنين امرى خواهد بود (TrT).

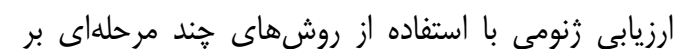

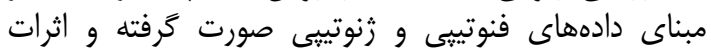

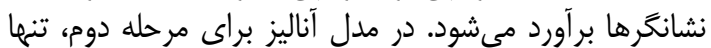

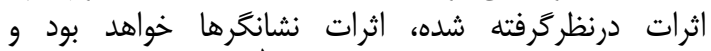

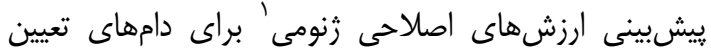

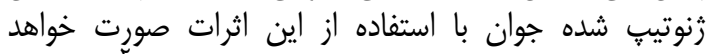

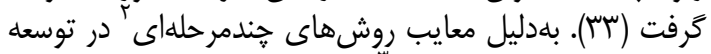

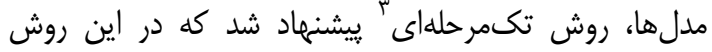

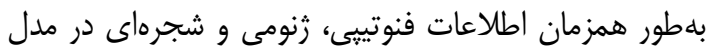

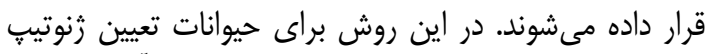

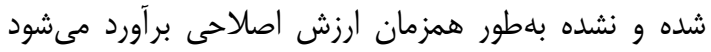

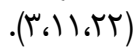

روشهاى SSBR' با تلفيق ماتريس روابط خويشاوندى

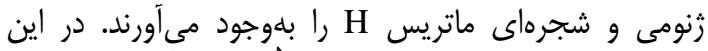

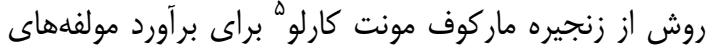

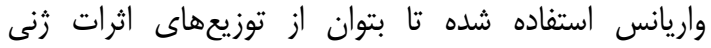
متنوعترى بجرهمند شد (1). بنابر نتايج يثوهش اندان انجام شده،

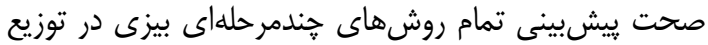

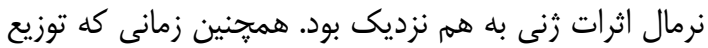

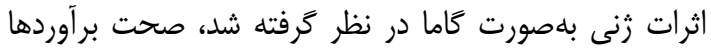

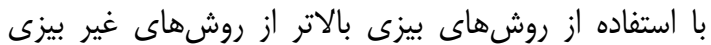

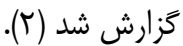
توزيع نرمال يكى از مهمترين توزئ توزيعهاى احتمالى ييوسته

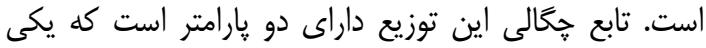




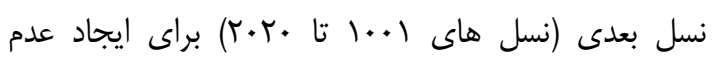

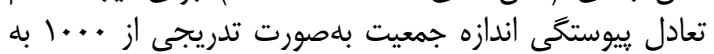

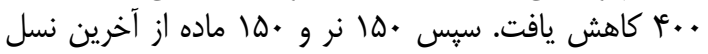

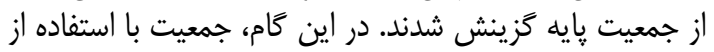

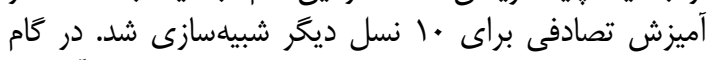

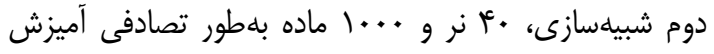

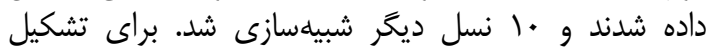

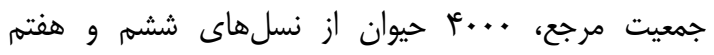

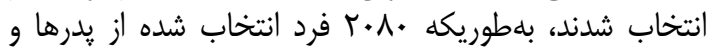

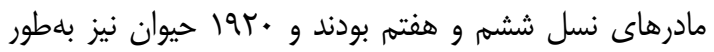

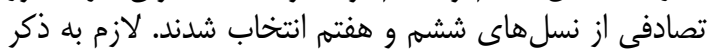

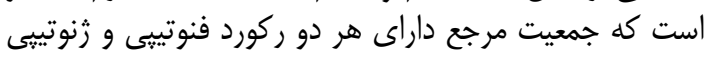

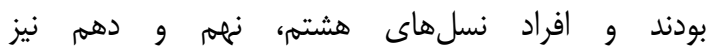

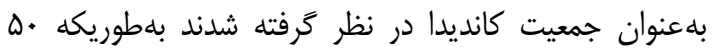

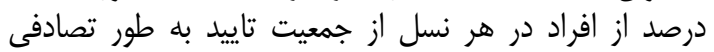

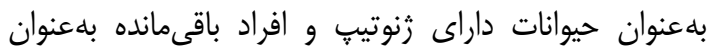

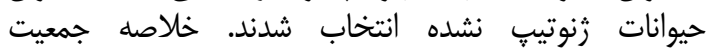

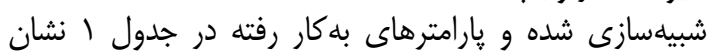

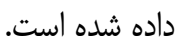

Table 1. Parameters of the simulation process
يزوهش حاضر با هدف بررسى صحت ارزيابى زنومى

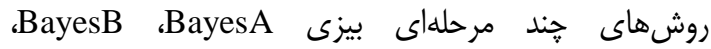

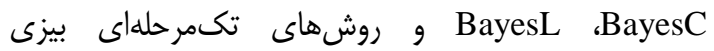
SSBR-A ور مقادير متفاوت $\pi$ SSBR-C

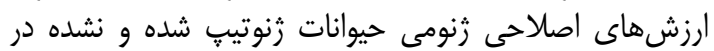

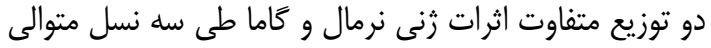
انجام شد.

\section{مواد و روشها \\ شبيهسازى زنوم روني}

جمعيتها با استفاده از نرمافزار

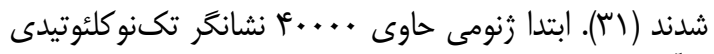

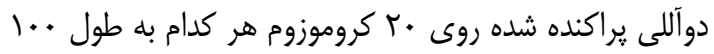

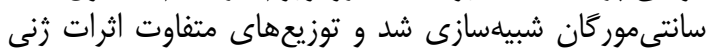

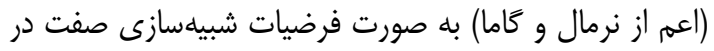

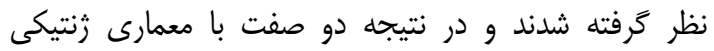

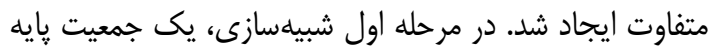

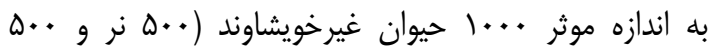

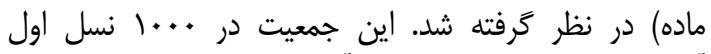

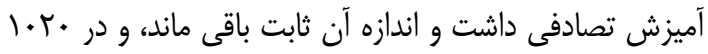

جدول 1 - يارامترهاى فرايند شبيهسازى

\begin{tabular}{|c|c|}
\hline جمعيت اول/دوم & ساختار جمعيت \\
\hline & جمعيت اوليه \\
\hline $1 \cdots(1 \cdots)$ & فاز اول تعداد نسل (تعداد افراد) \\
\hline \multirow[t]{2}{*}{$r \cdot r \cdot(r+)}$. & فاز دوم تعداد نسل (تعداد افراد) \\
\hline & جمعيت بسط داده شده \\
\hline 10 . & تعداد نرهاى از جمعيت بايه \\
\hline 10 . & تعداد مادههاى از جمعيت پايه \\
\hline 1. & تعداد نسل \\
\hline f. & تعداد نرهاى از جمعيت بسط داده شده \\
\hline $1 \ldots$ & تعداد مادههاى از جمعيت بسط داده شده \\
\hline 1. & تعداد نسل \\
\hline .10 . & احتمال نر بودن نتاج \\
\hline تصادفى & كزينش و طرح آميزش \\
\hline ارزش اصلاحى برآوردى & معيار حذف ل ل \\
\hline & رنوم \\
\hline r. & تعداد كروموزوم \\
\hline $1 .$. & طول هركروموزوم (سانتى موركان) \\
\hline f.... & تعداد نشانكرهاى تكنوكلئوتيدى \\
\hline$r \cdot$. & تعدادLQTL ها \\
\hline نرمال/ كاما (با پارامترشكل=ع/×•) & اثر الل هاى QTL \\
\hline$T / \Delta \times)^{-\infty}$ & نرخ جهش در نشانكَر و QTL ها \\
\hline$\cdot / \%$ & وراثتيذيرى - مي \\
\hline
\end{tabular}

نشانگرى و e اثر باقيمانده هستند. توزيع קُّالى احتمـال تـوام ييشين مجهولات رابطه فوق به صورت زير است: $\mathrm{P}\left(\mu, \mathrm{b}, \sigma^{2} \mid \mathrm{df}, \mathrm{s}, \omega\right) \sim$
$\left\{\prod_{\mathrm{j}=1}^{\mathrm{m}} \mathrm{P}\left(\left.\mathrm{b}_{\mathrm{j}}\right|_{, \sigma_{\mathrm{bj}}} ^{\sigma^{2}}\right) \mathrm{P}\left(\Theta_{\mathrm{bj}} \mid \omega\right)\right\} \mathrm{X}^{-2}\left(\sigma^{2} \mid \mathrm{df}, \mathrm{s}\right)$

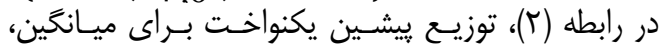

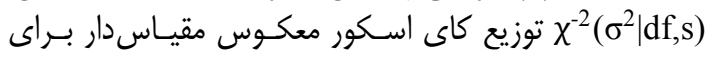

روش هاى جُندمرحلهاى بيزى

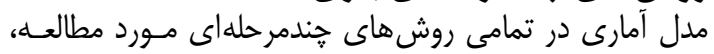

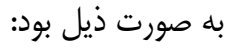
$\mathrm{y}_{\mathrm{i}}=\mu+\sum_{\mathrm{i}=1}^{\mathrm{m}} \mathrm{X}_{\mathrm{ij}} \mathrm{b}_{\mathrm{j}}+\mathrm{e}_{\mathrm{i}}$

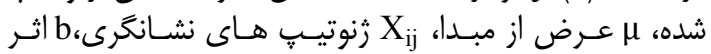




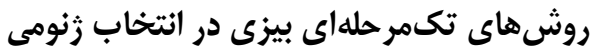

مدل روشهاى تكمر حلهاى بيزى بلهصورت زير برير بود:

$\mathrm{y}=\mathrm{X} \beta+\mathrm{ZM} \alpha+\mathrm{Z}_{\mathrm{n}} \varepsilon+\mathrm{e}$

در رابطه (ه)، ل بردار مشاهدات براى حيوانات تعيين ثنوتيب ، شده و ر نشده ، $\beta=\left[\begin{array}{l}\mu \\ \mu_{\mathrm{g}}\end{array}\right]$ ،

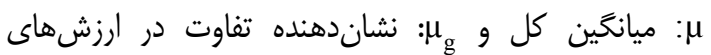
اصلاحى بين حيوانات زرنوتيب شده و نشده مى باشد. علاوه

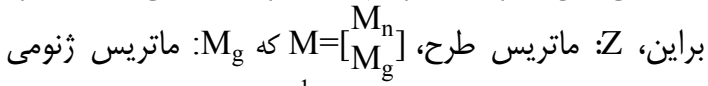

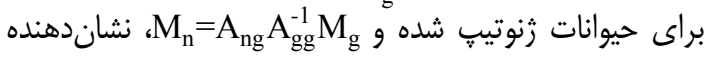

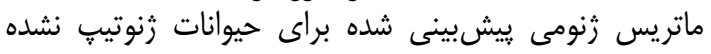

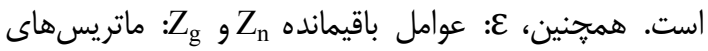

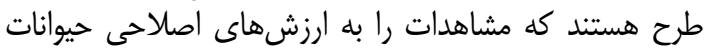

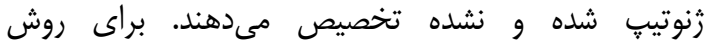

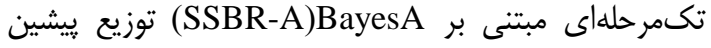

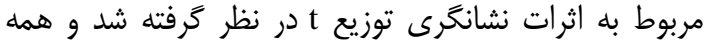

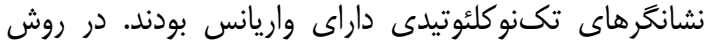

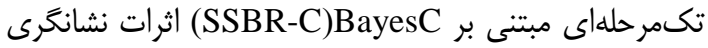
بلهورت زير در نظر كرفته شدند:

$\alpha_{\mathrm{j}} \mid \pi, \sigma_{\alpha \mathrm{j}}^{2} \sim \operatorname{IID}\left\{\begin{array}{c}0 \text { with probablity } \pi \\ \mathrm{N}\left(0, \sigma_{\alpha \mathrm{j}}^{2}\right) \text { with probablity } 1-\pi\end{array}\right.$

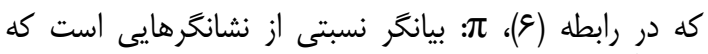

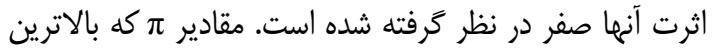

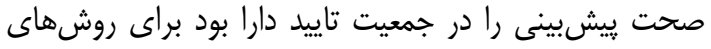
صدت

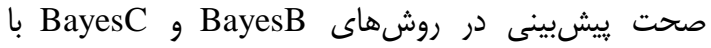

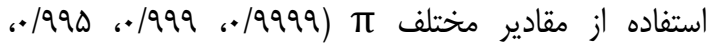

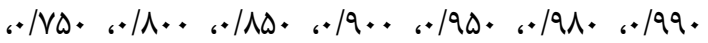

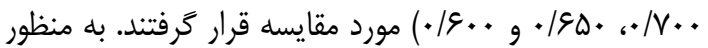

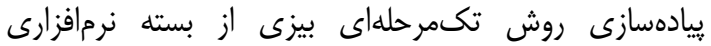

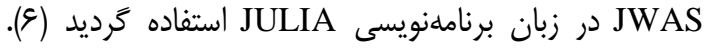

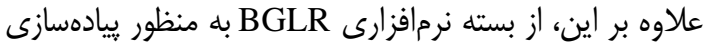

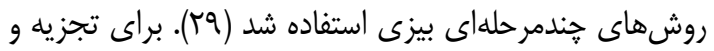

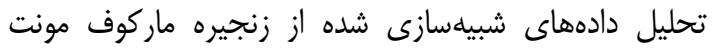

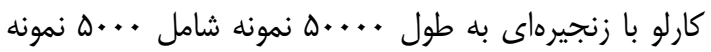

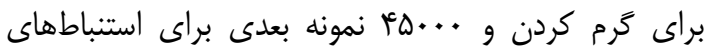

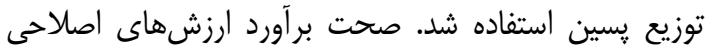

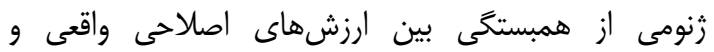

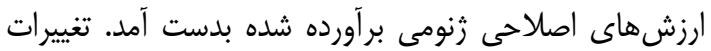

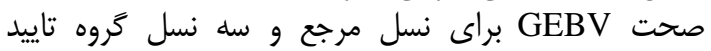

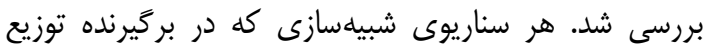

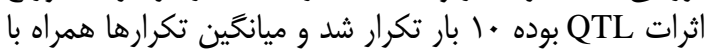
خطاى استاندارد ميانكَين ها محاسبه شدار تيد
واريانس باقى مانده با درجه آزادى df و هارامتر مقيـاس برابـر

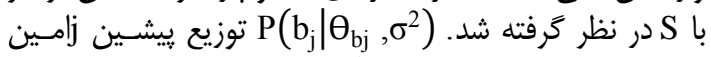

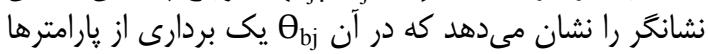

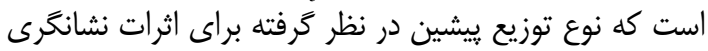

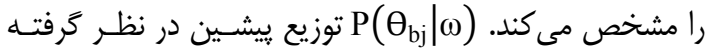

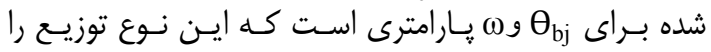

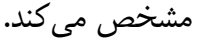

BayesA

در روش BayesA (سٓر) واريانس اثرات نشـانغرى متغيـر

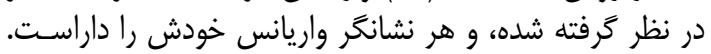

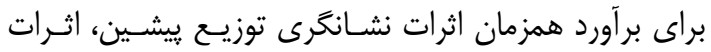

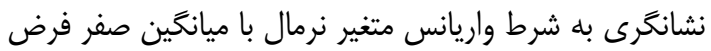

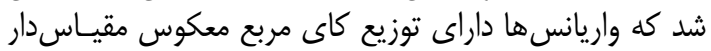

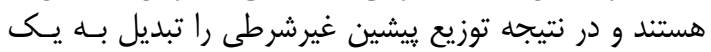

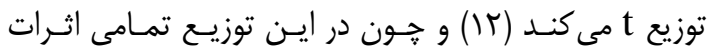

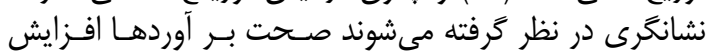

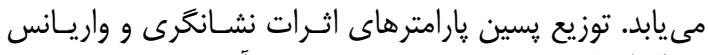

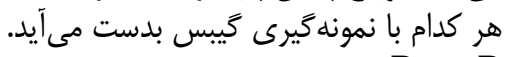

BayesB

در روش BayesB (זr) اثرات نشانكَرى با احتمال $\pi$ برابر با

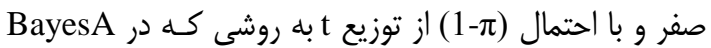
شرح داده شده به دست مى آيند. به اين صورت كه: آله

$b_{j} \mid d f_{b}, S_{b}, \pi \sim I I D\left\{\begin{array}{c}0 \text { with probablity } \pi \\ t\left(b_{i} \mid d f_{b}, S_{b}\right) \text { with probablity } 1\end{array}\right.$

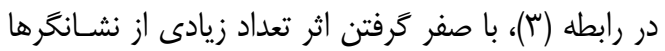

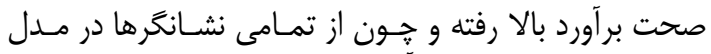

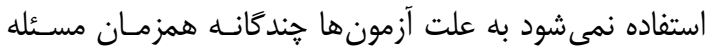

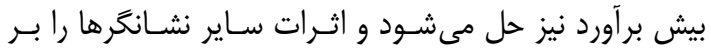
اساس توزيع ييشين منقبض مي كند مئد

BayesC

BayesB

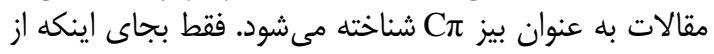

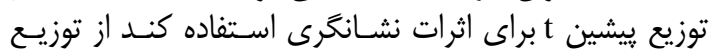

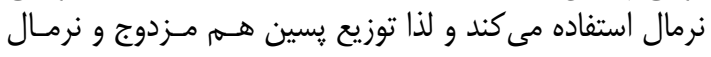

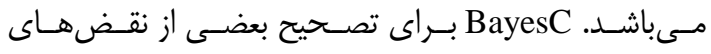

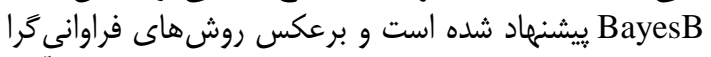

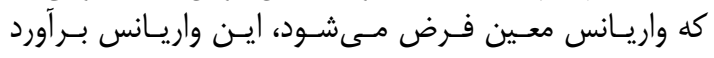

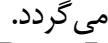
BayesL

در روش BayesL كه بيز 'LASSO هم ناميده مىشـود

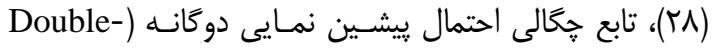

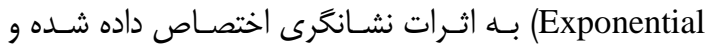
شكل آن به صورت زير است:

$\operatorname{DE}\left(\mathrm{b}_{\mathrm{j}} \mid \lambda, \sigma_{\mathrm{e}}^{2}\right)=\int \mathrm{N}\left(\mathrm{b}_{\mathrm{j}} \mid 0, \sigma_{\mathrm{e}}^{2} \tau_{\mathrm{j}}^{2}\right) \operatorname{EXP}\left(\tau_{\mathrm{j}}^{2} \mid \lambda^{2}\right) \tau_{\mathrm{j}}^{2}$

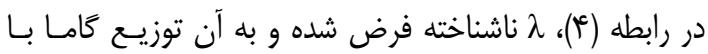

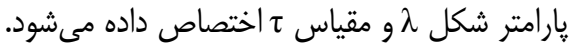


بلهورى كه صحتهاى بيشبينى شده با افزايش مقادير $\pi$ تا تا

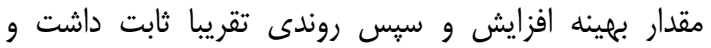

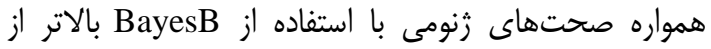
BayesC

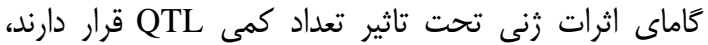

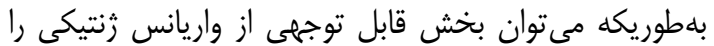

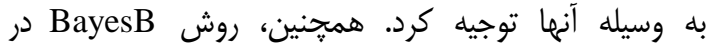

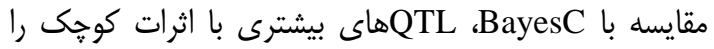
به صفر منقبض كرد، كه در نتيجه آن موجب بات بدام آندات انداختن

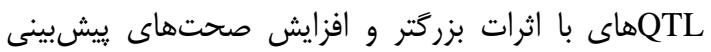

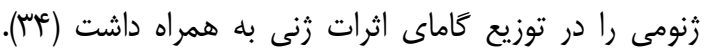

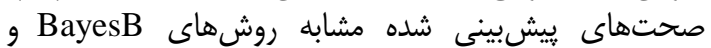
BayesC

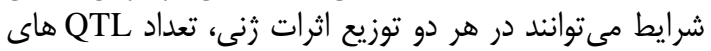
كم با اثرات بزرى را راتوجيه كنند.

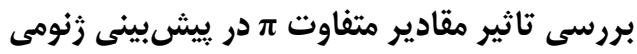

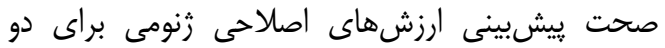

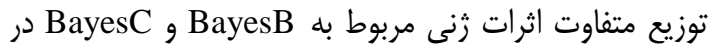

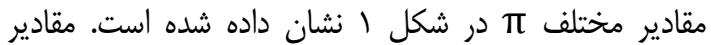

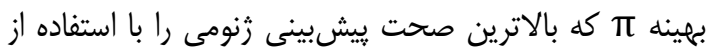

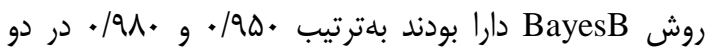

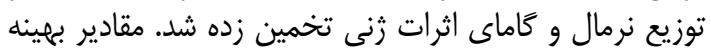

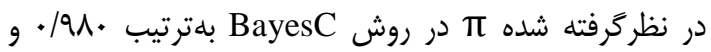

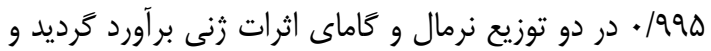

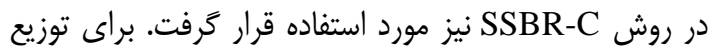

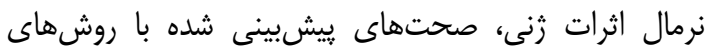

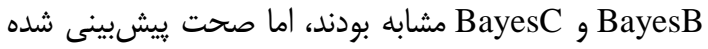

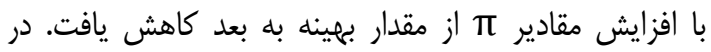
توزيع كَاماى اثرات زنى، الخَوى متفاوتى مشاهده كَرديد،

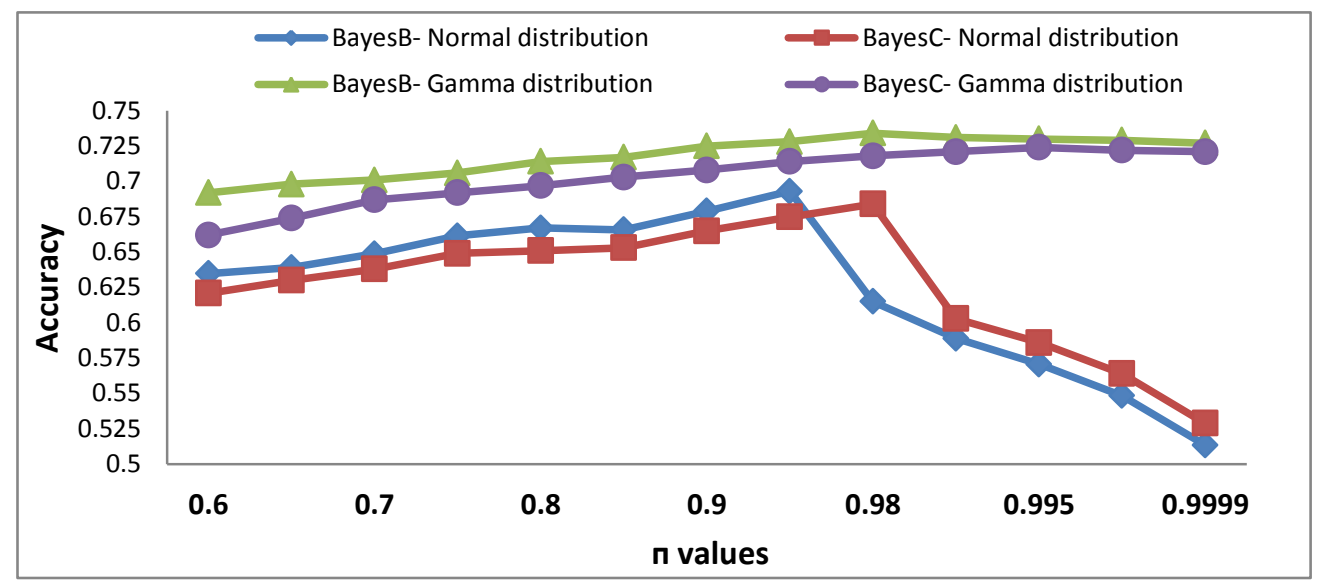

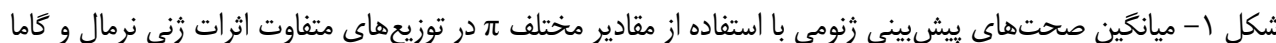

Figure 1. The mean prediction accuracies using different $\pi$ values in the normal and gamma distributions of QTL

و SSBR-C ( $\pi=0)$ ،SSBR-C ( $\pi=0.995)$ ،SSBR-A دSBR-C ( د Estimation) يش

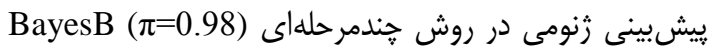

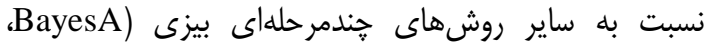
،BayesC $(\pi=0.995)$ ،BayesB ( $\pi$ Estimation) ز) (BayesL ،BayesC ( $\pi$ Estimation) ،BayesC $(\pi=0)$

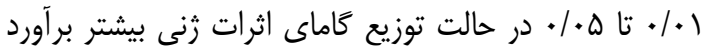

مقايسه روش هاى جند مر حلهاى و تكى مرحلهاى بيزى

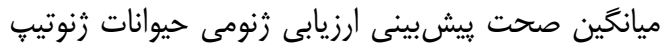

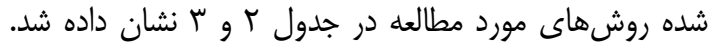

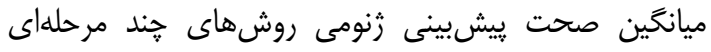
BayesB ( $\pi$ ، BayesB ( ،BayesC ( $\pi=0)$ ،BayesC $(\pi=0.995)$ ،Estimation) BayesL و BayesC ( $\pi$ Estimation) توزي

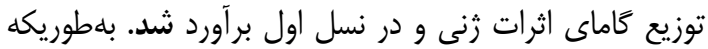

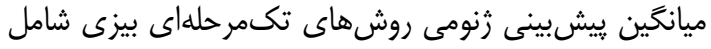


جدول r- ميانگين صحت (خطاى استاندارد) يِشبينى زنومى براى حيوانات زنوتيڤ شده در توزيع كاماى اثرات ثنها طى سه نسل متوالى Table 2. Meanof genomic prediction accuracies and standard errors (in parenthesis) for the genotyped animals in the gamma distribution of QTL effects during three consecutive generations

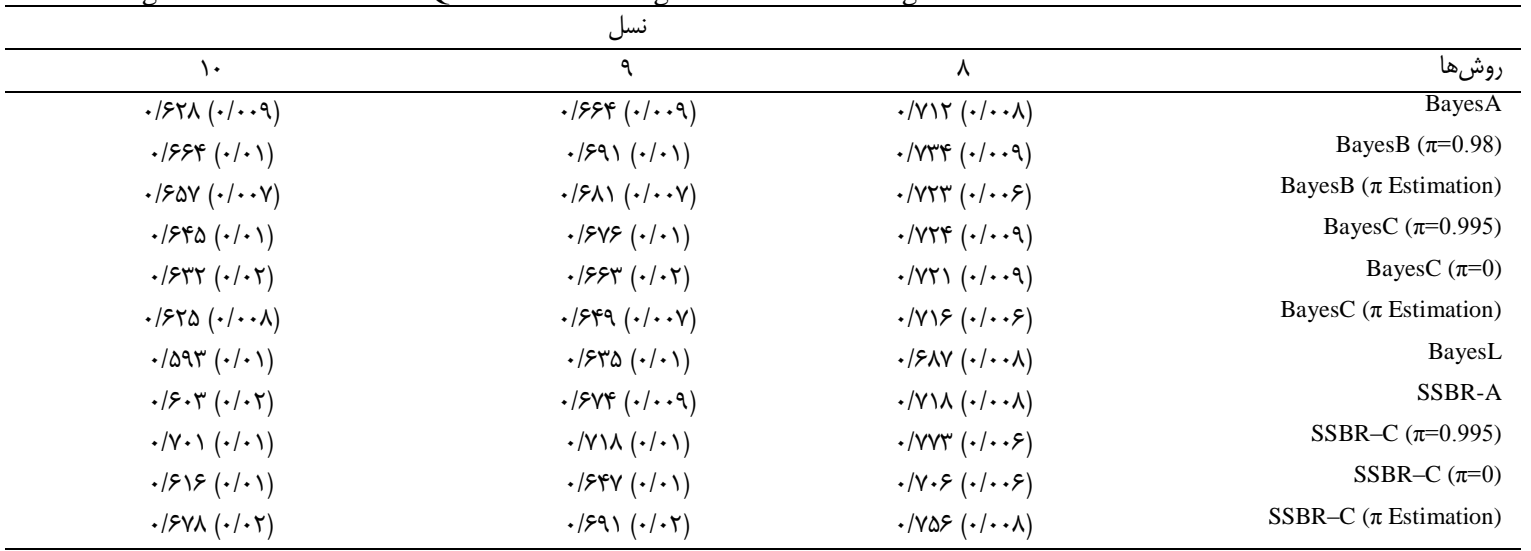

جدول س- ميانكَين صحت (خطاى استاندارد) ييشبينى زنومى براى حيوانات زنوتيٍ شده در توزيع نرمال اثرات ثنها طى سه نسل متوالى Table 3. Meanof genomic prediction accuracies and standard errors (in parenthesis) for the genotyped animals in the normal distribution of QTL effects during three consecutive generations

\begin{tabular}{|c|c|c|c|}
\hline \multicolumn{4}{|c|}{ نسل } \\
\hline 1. & 9 & $\wedge$ & روشها \\
\hline$\cdot / 9 \cdot r(\cdot / \cdot .9)$ & $\cdot / 9 r q(\cdot / \cdot \cdot V)$ &.$/ 991(\cdot / . .9)$ & BayesA \\
\hline.$|94|(\cdot / \cdot \cdot 1)$ &. $\operatorname{lgrv}(\cdot / \cdot \cdot 1)$ & $.894(\cdot / .99)$ & BayesB $(\pi=0.95)$ \\
\hline $.1919(\cdot 1 . .9)$ &.$/ 9 \mu f(\cdot / . . q)$ & $\cdot \mid \& \wedge \varepsilon(\cdot / \cdot 1)$ & BayesB ( $\pi$ Estimation) \\
\hline$\cdot / 8 \cdot v(\cdot / \cdot \cdot 1)$ &.$/ 9 T \Delta(\cdot / \cdot \cdot 1)$ &.$/ 9 \Lambda \mathrm{r}(\cdot / \cdot \cdot \mathrm{V})$ & BayesC $(\pi=0.98)$ \\
\hline $.1094(\cdot / . .9)$ & $\cdot\left|g_{\mu}\right|(\cdot / \cdot V)$ &.$/ 8 \wedge 9(\cdot / \cdot . \mu)$ & BayesC $(\pi=0)$ \\
\hline$\cdot \operatorname{loAr}(\cdot / \cdot 1)$ &.$/ 9 r g(. / . .9)$ & $.19 \vee 9(\cdot / . .9)$ & BayesC ( $\pi$ Estimation) \\
\hline.$/ 814(\cdot / . .9)$ & $\cdot \mid g_{T K}(\cdot / \cdot V V)$ &.$/ 9 \vee 8(. / . .9)$ & BayesL \\
\hline$\cdot / 9 \cdot v(\cdot 1 \cdot 1)$ & . & $\cdot / v \cdot r(\cdot / \cdot \cdot \Lambda)$ & SSBR-A \\
\hline.$/ \& \Gamma \Delta(\cdot / \cdot 1)$ & $.1994(.1 .1)$ & $\cdot / V \cdot 9(\cdot / \cdot .9)$ & SSBR-C $(\pi=0.98)$ \\
\hline$\cdot \operatorname{lgrg}(\cdot / \cdot \cdot 1)$ &.$\left|\varnothing_{\Delta}\right|(\cdot / \cdot \cdot 1)$ & $.1991(\cdot / . .9)$ & $\operatorname{SSBR}-\mathrm{C}(\pi=0)$ \\
\hline.$/$ gre $(\cdot / \cdot 1)$ &.$/ \operatorname{sov}(\cdot / \cdot 1)$ & $\cdot / V \cdot r(\cdot / \cdot N)$ & SSBR-C ( $\pi$ Estimation) \\
\hline
\end{tabular}

از ركورد حيوانات تعيين زنوتيڤ شده و نشده بلطفور همزمان

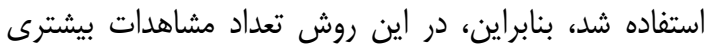

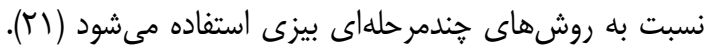

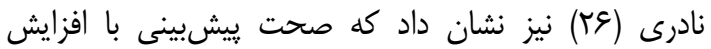

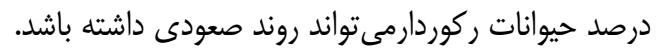

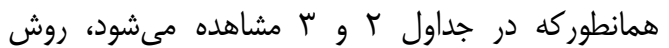
SSBR-C ( $(\pi=0.995)$ (•/VYr) SSBR-C (

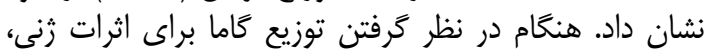

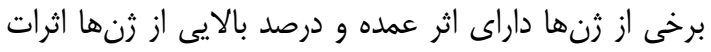

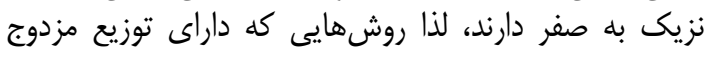

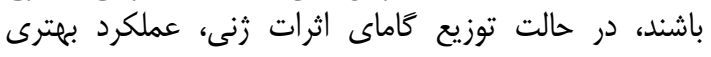

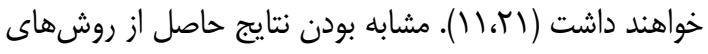

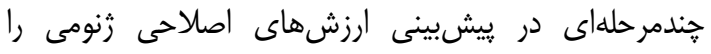

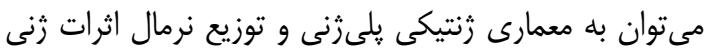

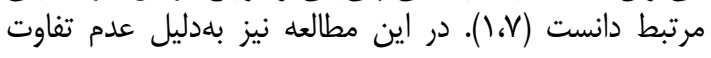

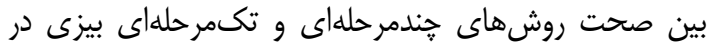

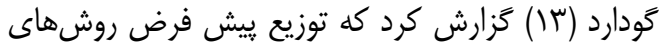

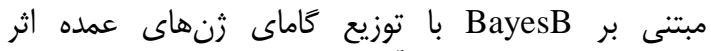

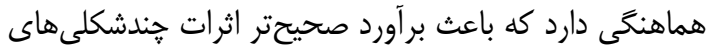

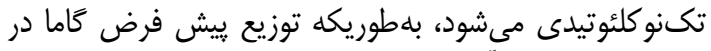

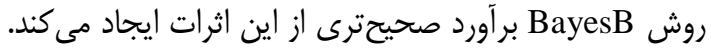

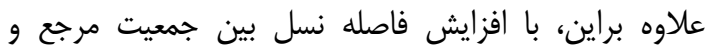

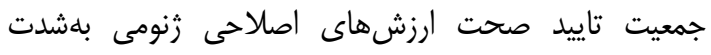

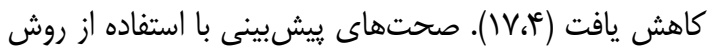

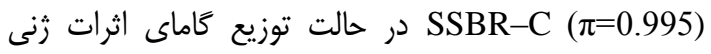

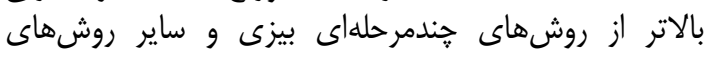

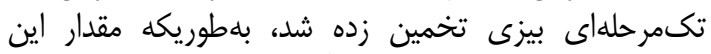

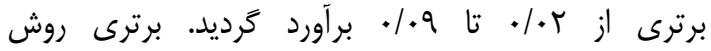
تكمرحلهاى SSBR-C (

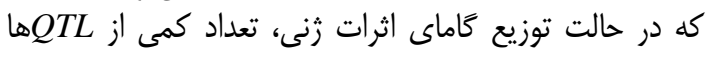

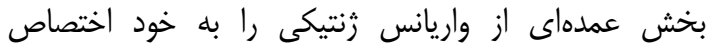

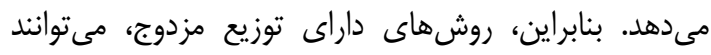

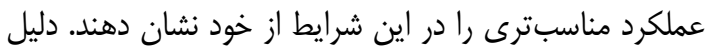

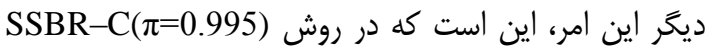


تاثير روابط خويشاوندى شجرهاى و زنومى بين جمعيت مرجع و تاييد

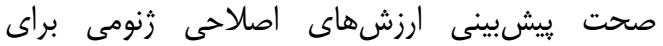

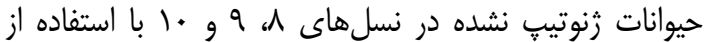

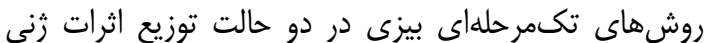

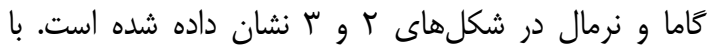

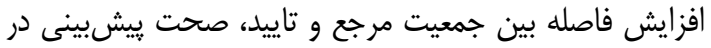

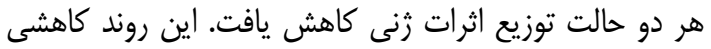

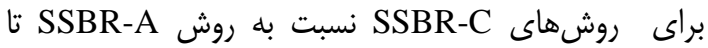

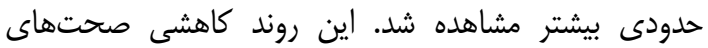

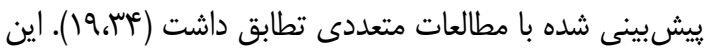

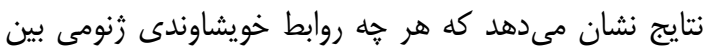

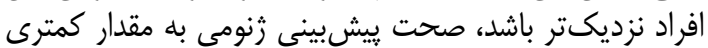

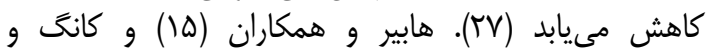

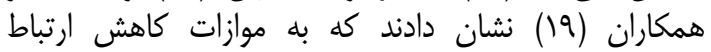

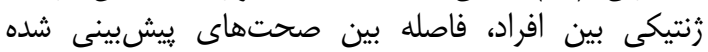

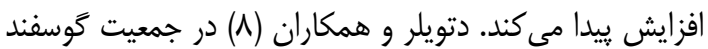

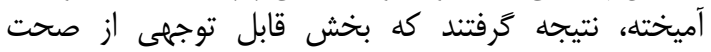

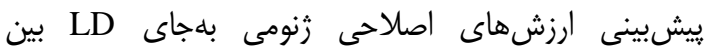

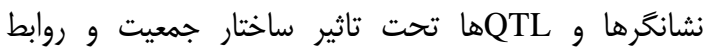

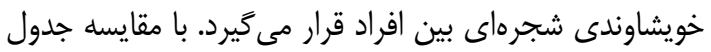

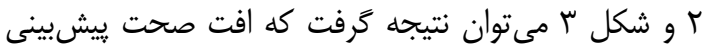

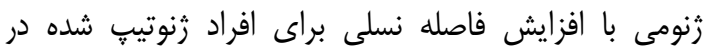

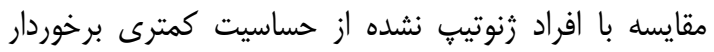

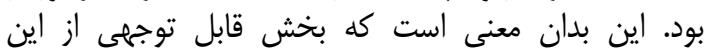

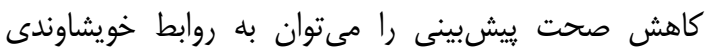

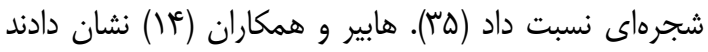

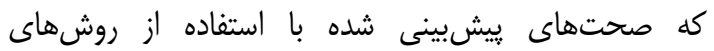

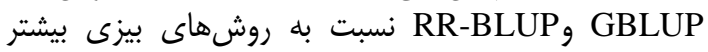
تحت تاثير روابط خويشاوندى زنتيكى اعم از شجرهاى

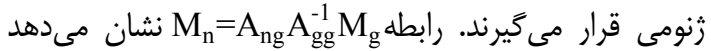

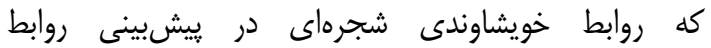

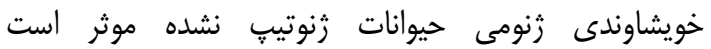

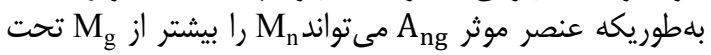

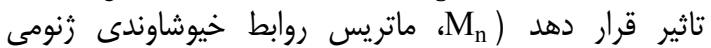

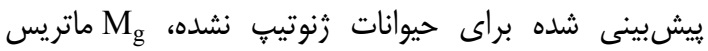

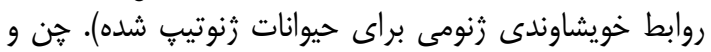

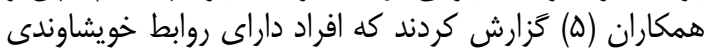

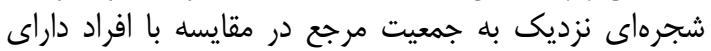

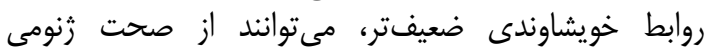
ييشبينى شده بالاترى برخوردار باشند. نتايج اين يثروهش

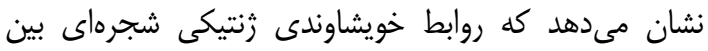

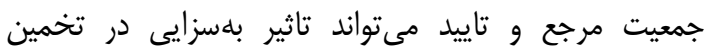

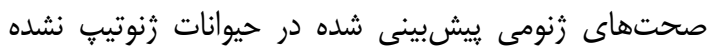

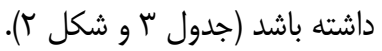

حالت توزيع نرمال اثرات زنى (جدول ऍّ) و با توجه به توانايى

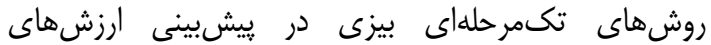

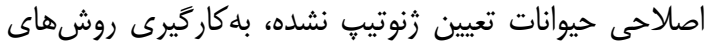

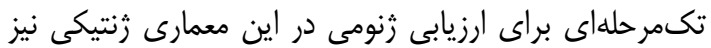

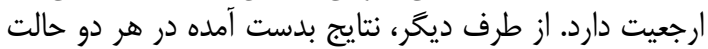

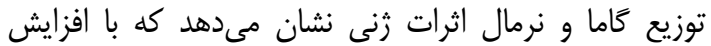

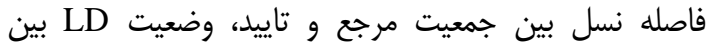

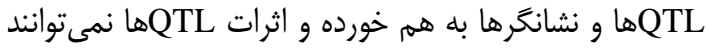

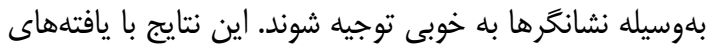

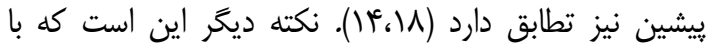

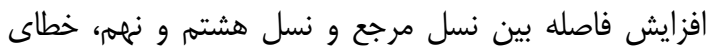

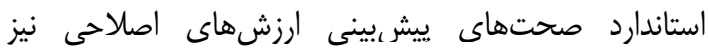

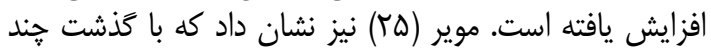

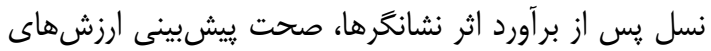

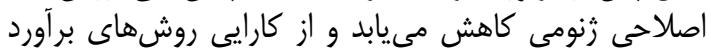

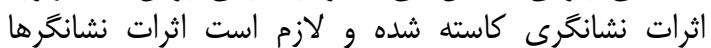

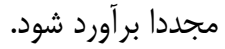

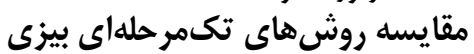

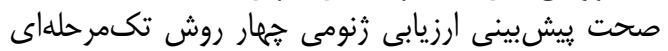
SSBR-C ، SSBR-C ( SSBR-C ( $\pi$ Estimation) ور حالت توزيع كاما و

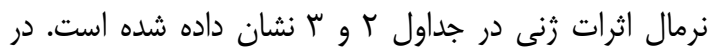
حSBR-C ( Estimation) را نسبت به SSBR-A از خود نشان دادند بهطورى كه اين

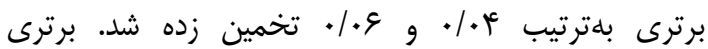

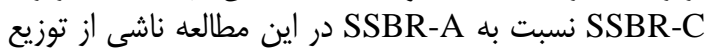
مزدوجى است كه در روش SSBR-C مورد استفاده قرار

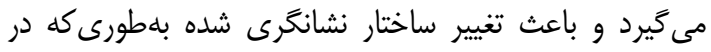

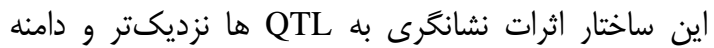

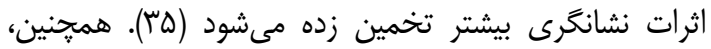

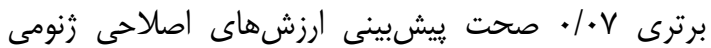
توسط روش SSBR-C

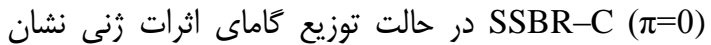
مىدهد كه در نظر گرفتن توزيع مزدوج در اين شرايط مئ توتواند

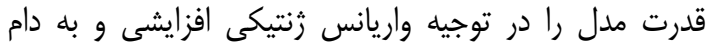

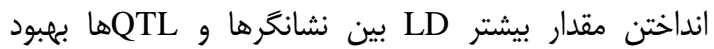

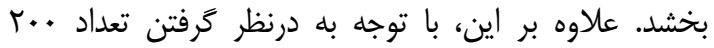

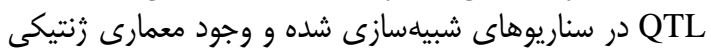

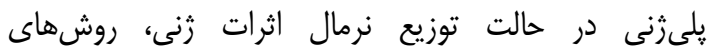

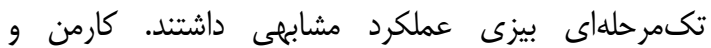

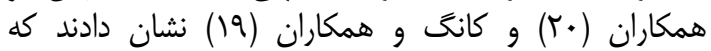

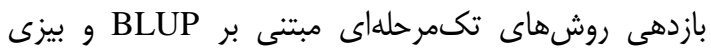

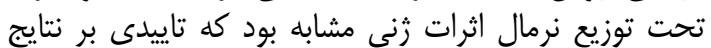
تحقيق حاضر است. تونت 


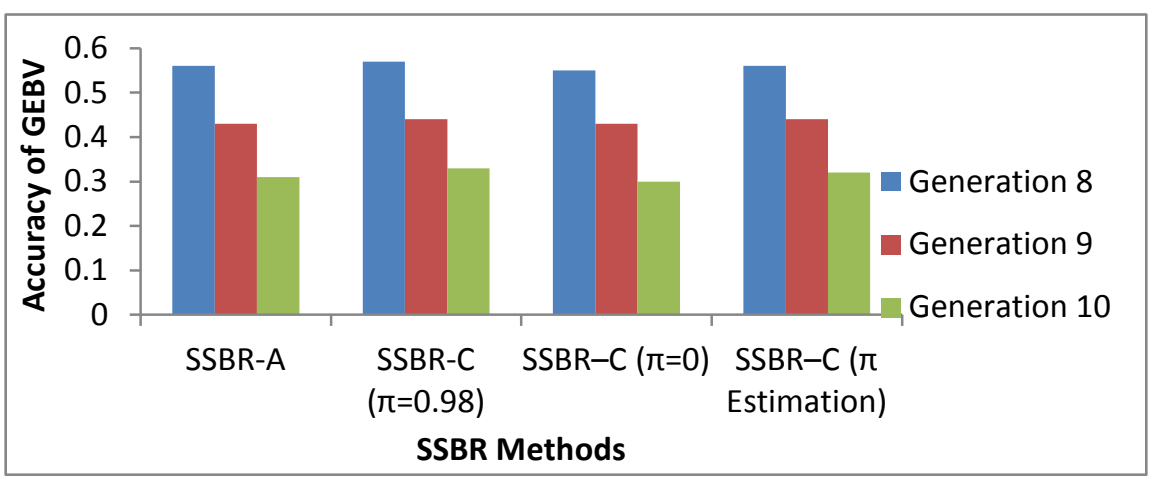

شكل r- ميانگين صحت يِشبينى ارزشهاى اصلاحى زنومى با استفاده از روشهاى تكىمرحلهاى بيزى براى حيوانات زنوتيب نشده مربوط

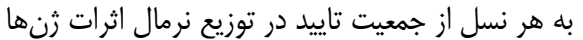

Figure 2. Mean of genomic prediction accuracies using single step Bayesian regression for the nongenotyped animals for each validation set in the normal distribution

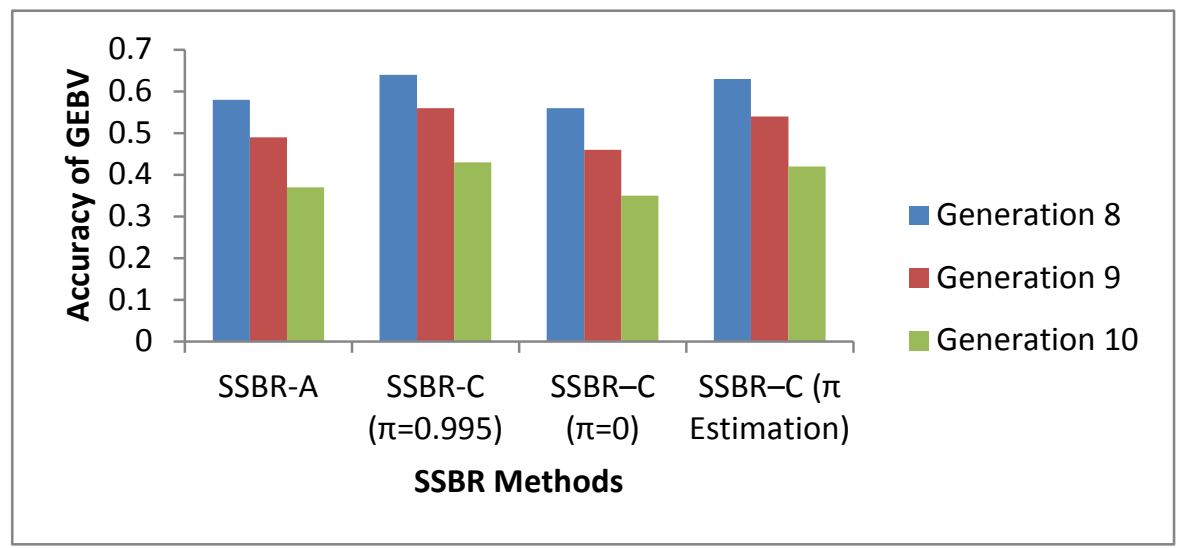

شكل ؟- ميانكَين صحت يِشبينى ارزشهاى اصلاحى زنومى با استفاده از روشهاى تكىمرحلهاى بيزى براى حيوانات زنوتيب نشده مربوط

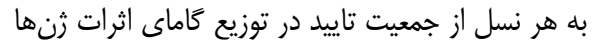

Figure 3. Mean of genomic prediction accuracies using single step Bayesian regression for the nongenotyped animals for each validation set in the gamma distribution

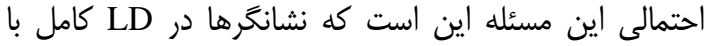

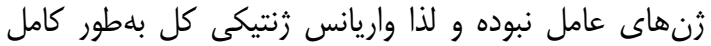

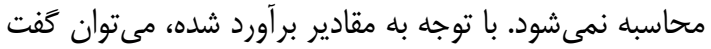

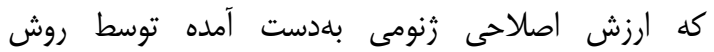
SSBR-C ( $\pi=0)$

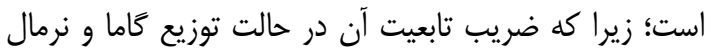

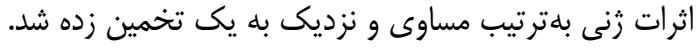

ميانگين ضريب تابعيت ارزشهاى اصلاحى واقعى به ارزشهاى اصلاحى تخمين زده شده، در جدول أ نشان داديت داده

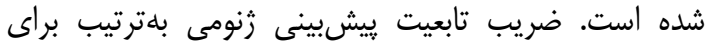

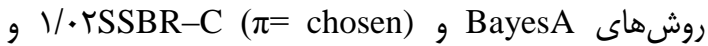

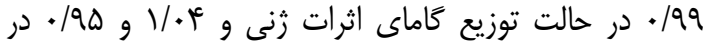

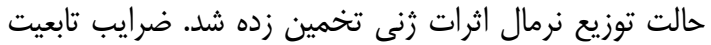

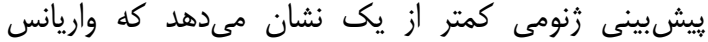
ارزشهاى اصلاحى زنومى بيش از حد بر برآورد شده است. دليل دهي 
جدول عا ضريب تابعيت (خطاى استاندارد) ارزش هاى اصلاحى واقعى به ارزشهاى اصلاحى تخمين زده شده در توزيع اثرات زنى نرمال و كاما Table 4. Regression and standard error (in parenthesis) of true on estimated breeding value in the distribution of normal and gamma QTL effects

\begin{tabular}{|c|c|c|}
\hline \multicolumn{2}{|c|}{ توزيع اثرات زنى } & روشها \\
\hline نرمال & كاما & \\
\hline$Y / \cdot F(\cdot / \cdot r)$ & $\lambda / \cdot r(\cdot / \cdot r)$ & BayesA \\
\hline $1 / \cdot r(\cdot / \cdot r)$ & $M \cdot t(\cdot / \cdot r)$ & BayesB $(\pi=\text { chosen })^{\mathrm{a}}$ \\
\hline $1 / \cdot \Delta(\cdot / \cdot r)$ & $V \cdot r(\cdot / \cdot r)$ & BayesB ( $\pi$ Estimation) \\
\hline $1 / \cdot c(\cdot / \cdot r)$ & I/.r $(\cdot / \cdot r)$ & BayesC $(\pi=\text { chosen })^{\mathrm{D}}$ \\
\hline $1 / \cdot r(\cdot / \cdot r)$ & $1 / \cdot(\cdot / \cdot r)$ & BayesC $(\pi=0)$ \\
\hline $1 / \cdot \Delta(\cdot 1 \cdot 1)$ & $1 / \cdot r(\cdot / \cdot 1)$ & BayesC ( $\pi$ Estimation) \\
\hline $1 / \cdot \Delta(\cdot / \cdot r)$ & $1 / \mu(\cdot / \cdot r)$ & BayesL \\
\hline $1 / \cdot r(\cdot / \cdot r)$ & $1 / .9(\cdot 1 \cdot 1)$ & SSBR-A \\
\hline$\cdot / 90(\cdot / \cdot r)$ &.$/ 99(\cdot / \cdot r)$ & $\operatorname{SSBR}-\mathrm{C}(\pi=$ chosen $)$ \\
\hline.$/ 99(\cdot / \cdot r)$ & $1 / \cdot(\cdot / \cdot r)$ & $\operatorname{SSBR}-\mathrm{C}(\pi=0)$ \\
\hline.$/ 91(\cdot 1 \cdot 1)$ &.$/ 99(\cdot / \cdot r)$ & SSBR-C ( $\pi$ Estimation) \\
\hline
\end{tabular}

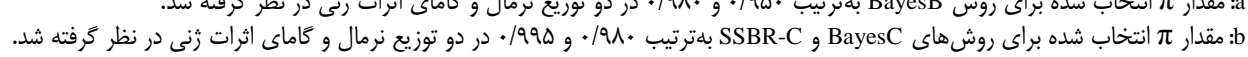

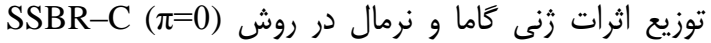

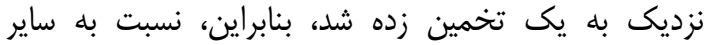

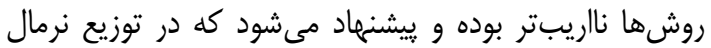

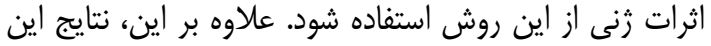

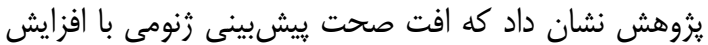

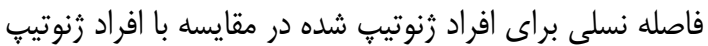
نشده از حساسيت كمترى برخوردار بود.

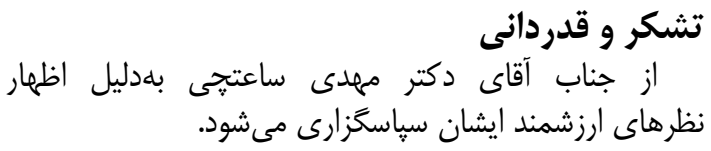

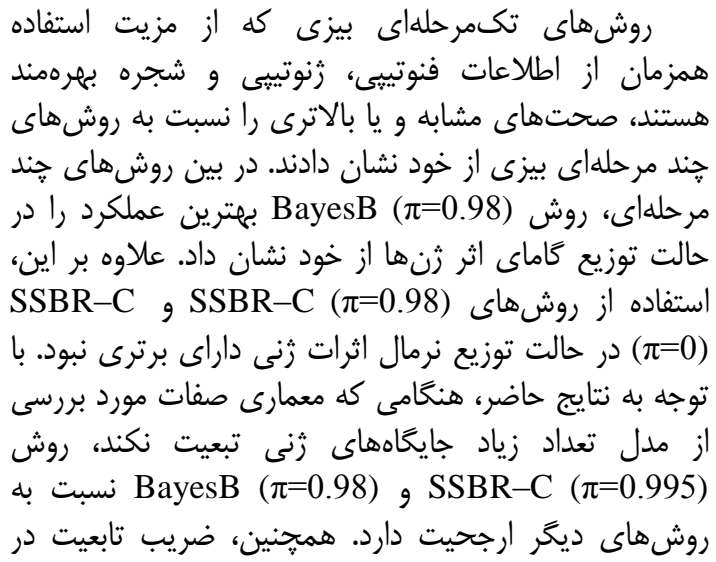

1. Abdollahi-Arpanahi, R. 2013. The Impact of allelic architecture of complex traits on genetic evaluations and evolutionary genetics. Ph.D. Dissertation University of Tehran, Tehran.

2. Abdollahi-Arpanahi, R., A. Pakdel, A. Nejati-Javaremi and M.M. Shahrbabak. Comparison ofdifferent methods of genomic evolution in traits with different genetic architecture. Journal of Animal Production, 15: 65-77 (In Persian).

3. Aguilar, I., I. Misztal, D.L. Johnson, A. Legarra and S. Tsuruta. 2010. Hot topic: A unified approach to utilize phenotypic, full pedigree, and genomic information for genetic evaluation of Holstein final score. Journal of Dairy Science, 93: 743-752.

4. Calus, M.P. 2009. Genomic breeding value prediction: methods and procedures. Animal, 4(2): 157-164.

5. Chen, L, C. Li, M. Sargolzaei and F. Schenkel. 2014. Impact of genotype imputation on the performance of GBLUP and Bayesian methods for genomic prediction. PLoS ONE, 9(7): e101544.

6. Cheng, H., D.J. Garrick and R.L. Fernando. 2016. JWAS: Julia implementation of whole-genome analyses software using univariate and multivariate Bayesian mixed effects model. Retrieved June 8, 2019 from http://QTL.rocks.

7. Colombani, C., A. Legarra, S. Fritz, F. Guillaume, P. Croiseau, V. Ducrocq and C. Robert-Granié. 2012. Application of Bayesian least absolute shrinkage and selection operator (LASSO) and BayesCp methods for genomic selection in French Holstein and Montbéliarde breeds. Journal of Dairy Science, 96: 575-91.

8. Daetwyler, H.D., K.E. Kemper, J.H. Vander Werf and B.J. Hayes. 2012. Components of the accuracy of genomic prediction in a multi-breed sheep population, Journal of Animal Science, 90(10): 3375-3384.

9. De los Campos, G., H. Naya, D. Gianola, J. Crossa, A. Legarra, E. Manfredi, K. Weigel and J.M. Cotes. 2009. Predicting quantitative traits with regression models for dense molecular markers and pedigree. Genetics, 182(1): 375-385.

10. De los Campos, G., A.I. Vazquez, R. Fernando, Y.C. Klimentidis and D. Sorensen. 2013. Prediction of complex human traits using the genomic best linear unbiased predictor. PLoS Genetics, 9: e1003608. 
$1 \%$. مقايسه روشهاى جند مرحلهاى و تكى مرحلهاى بيزى

11. Fernando, R.L., J.C. Dekkers and D.J. Garrick. 2014. A class of Bayesian methods to combine large numbers of genotyped and non-genotyped animals for whole-genome analyses. Genetics Selection Evolution, 46: 50.

12. Gianola, D., G. DeLos Campos, W.G. Hill, E. Manfredi and R. Fernando. 2009. Additive genetic variability and the Bayesian alphabet. Genetics, 183: 347-363.

13. Goddard, M. 2009. Genomic selection: prediction of accuracy and maximization of long term response. Genetica, 136: 245-257.

14. Habier, D., R.L. Fernando, J.C. Dekkers. 2007. The impact of genetic relationship information on genomeassisted breeding values. Genetics, 177(4): 2389-2397.

15. Habier, D., J. Tetens, F.R. Seefried, P. Lichtner and G. Thaller. 2010. The impact of genetic relationship information on genomic breeding values in German Holstein cattle. Genetics Selection Evolution, 42(1): 5.

16. Habier, D., R.L. Fernando, K. Kizilkaya and D.J. Garrick. 2011. Extension of the Bayesian alphabet for genomic selection. BMC Bioinformatics, 2: 186-194.

17. Hayes, B.J., P.J. Bowman, A.J Chamberlain and M.E. Goddard. 2009. Invited review: Genomic selection in dairy cattle: Progress and challenges. Journal of DairyScience, 92: 433-443.

18. Hickey, J., S. Dreisigacker, J. Crossa, S. Hearne, R. Babu, B. Prasanna, M. Grondona, A. Zambelli, V. Windhausen, K. Mathews and G. Gorjanc. 2014. Evaluation of genomic selection training population designs and genotyping strategies in plant breeding programs using simulation. Crop Science, 54: 14761488 .

19. Kang, H., L. Zhou, R. Mrode, Q. Zhang and J.F. Liu. 2016. Incorporating single-step strategy into random regression model to enhance genomic prediction of longitudinal trait. Heredity, 119: 459-467.

20. Karaman, E., H. Cheng, M.Z. Firat, D.J. Garrick and R.L. Fernando. 2016. An upper bound for accuracy of prediction using GBLUP. PLoS ONE, 11(8): e0161054.

21. Lee, J., H. Cheng, D. Garrick, B. Golden, J.C. Dekkers, K. Park, D. Lee and R. Fernando. 2017. Comparison of alternative approaches to single - trait genomic prediction using genotyped and non genotyped Hanwoo beef cattle. Genetiscs Selection Evolution, 49: 2.

22. Legarra, A., I. Aguilar and I. Misztal. 2009. A relationship matrix including full pedigree and genomic information. Journal of Dairy Science, 92: 4656-63.

23. Meuwissen, T., B. Hayes and M. Goddard. 2001. Prediction of total genetic value using genome-wide dense marker maps. Genetics, 157: 1819-29.

24. Moschopoulos, P.G. 1985. The distribution of the sum of independent gamma random variables. Annals of the Institute of Statistical Mathematics, 37(1): 541-544.

25. Muir, W.M. 2007. Comparison of genomic and traditional BLUP-estimated breeding value accuracy and selection response under alternative trait and genomic parameters. Journal of Animal Breeding and Genetics, 124: 342-355.

26. Naderi, Y. 2018. The importance of genetic relationships and phenotypic record on genomic accuracy of simulated imputation data via animal models in presence of genotype $\times$ environment interactions. Research on Animal Production, 22: 119-130 (In Persian).

27. Nejati-Javaremi, A., C. Smith and J. Gibson. 1997. Effect of total allelic relationship on accuracy of evaluation and response to selection. Journal of animal science, 75: 1738-45.

28. Park, T. and G. Casella. 2008. The Bayesian lasso. Journal of the American Statistical Association, 103: 681-6.

29. Pérez, P. and G. Delos Campos. 2014. Genome-wide regression and prediction with the BGLR statistical package. Genetics, 198: 483-495.

30. Saheb Alam, H., M. Gholizadeh, H. Hafezian and A. Farhadi. 2017. Comparison of Bayesian methods in the genomic evaluation with different genetic architecture. Research on Animal Production, 18: 177-186 (In Persian).

31. Sargolzaei, M. and F.S. Schenkel. 2009. QMSim: a large-scale genome simulator for livestock. Bioinformatics, 25: 680 .

32. Toghiani, S., S.E. Aggrey and R. Rekaya. 2016. Multi-generational imputation of single nucleotide polymorphism marker genotypesand accuracy of genomic selection. Animal, 10: 1077-85.

33. VanRaden, P.M., C.P. Van Tassell, G.R. Wiggans, T.S. Sonstegard, R.D. Schnabel, J.F. Taylor and F.S. Schenkel. 2009. Invited review: reliability of genomic predictions for North American Holstein bulls. Journal of Dairy Science, 92: 16-24.

34. Wolc, A., J. Arango, P. Settar, J.E. Fulton, N.P. O’Sullivan and J.C. Dekkers. 2016. Mixture models detect large effect QTL better than GBLUP and result in more accurate and persistent predictions. Journal of Animal Science, 7: 7.

35. Zhou, L., R. Mrode, S. Zhang, Q. Zhang, B. Li and J. Liu. 2018. Factors affecting GEBV accuracy with single-step Bayesian models. Heredity, 120: 100-109. 


\title{
Comparison of Single and Multi-Step Bayesian Methods for Predicting Genomic Breeding Values in Genotyped and Non-Genotyped Animals-A Simulation Study
}

\author{
Mostafa Madad Jirandeh ${ }^{1}$, Jalil Shodia ${ }^{2}$, SadeghAlijani ${ }^{3}$, Seyed Abbas Rafat ${ }^{2}$ and \\ Jack C.M. Dekkers ${ }^{4}$ \\ 1- Ph.D. Student, Department of Animal Sciences, University of Tabriz, Tabriz, Iran \\ 2- Professor, Department of Animal Sciences, University of Tabriz, Tabriz, Iran. \\ 3- Associate Professor, Department of Animal Sciences, University of Tabriz, Tabriz, Iran \\ (Corresponding author: sad-ali@ tabrizu.ac.ir) \\ 4- Professor, Department of Animal Sciences, Iowa state university, Ames, United States of America \\ Received: July 2, $2019 \quad$ Accepted: September 11, 2019
}

\begin{abstract}
The purpose of this study was to compare the accuracy of genomic evaluation for Bayes A, Bayes B, Bayes C and Bayes L multi-step methods and SSBR-C and SSBR-A single-step methods in the different values of $\pi$ for predicting genomic breeding values of the genotyped and non-genotyped animals. A genome with 40000 SNPs on the 20 chromosom was simulated with the same distance $(100 \mathrm{cM})$. The $\pi$ values that maximized the prediction accuracies in BayesC were 0.980 and 0.995 for the normal and gamma distributions of QTL, respectively, and were also used in SSBR-C method. Genomic prediction accuracy in the SSBR-C $(\pi=0.99)$ method was higher than multi step methods from 0.02 to 0.09 for gamma distribution. Results showed that considering mixture distribution and use of phenotype, genotype and pedigree information simultaneously, the SSBR-C $(\pi=0.99)$ method had higher accuracy than other methods and is considered a better choice in this scenario. Moreover, both single and multi-step methods showed similar prediction accuracy when the genetic architecture appeared to approach the normal distribution. Furthermore, SSBR-C $(\pi=0)$ method appeared to be more reliable choice that was due to regressions of true breeding value on estimated breeding value close to one in normal distribution. Generally, GEBV accuracy decreased as the distance increased between validations and training set, which was more sensitive for non-genotyped individuals compared to genotyped individuals.
\end{abstract}

Keywords: Bayesian, Genomic Accuracy, Genomic Selection, Multi-Step Methods, Simulation 